\title{
Téoros
}

Revue de recherche en tourisme

\section{Les fêtes populaires : héritage fécond et avenir prometteur}

\section{Pierre-Paul Leduc}

Volume 5, numéro 2, juillet 1986

Tourisme, fêtes et événements

URI : https://id.erudit.org/iderudit/1080569ar

DOI : https://doi.org/10.7202/1080569ar

Aller au sommaire du numéro

Éditeur(s)

Université du Québec à Montréal

ISSN

0712-8657 (imprimé)

1923-2705 (numérique)

Découvrir la revue

Citer cet article

Leduc, P.-P. (1986). Les fêtes populaires : héritage fécond et avenir prometteur. Téoros, 5(2), 5-6. https://doi.org/10.7202/1080569ar d'utilisation que vous pouvez consulter en ligne.

https://apropos.erudit.org/fr/usagers/politique-dutilisation/ 


\section{Les fêtes populaires: héritage fécond et avenir prometteur}

par Pierre-Paul Leduc*

Ce sont les. Français venus en Amérique qui ont fait revivre au Québec leurs fëtés dès les débuts de la colonié. Ils arrivèrent avec leurs rites et leurs fêtes religieuses datant du Moyen Àge.

D'ailleurs, nos fêttes d'aujourd'hui gardent encore des traces d'origine médievale: grandes messes, benédictions, arrêts à l'église, processions

Nos grandes fêtes sont, à majorité, un héritage reçu. La transformation de leurs rites et leur adaptation à la vie modeme sont très récentes et ont duré à bien des égards jusqu'à la fin des années soixante.

La fête populaire au Québec, en plus d'avoir un héritage riche et fécond, a pris de l'ampleur ces dernières années grâce à des interventions orientées à cette fin. Particulièrement depuis 1973, l'objectif poursuivi par les différents paliers du gouvernement et par tous les groupes concernés a été de faire la promotion de la fête populaire. Un programme d'assistance financière a été offert aux municipalités et aux organismes privés à but non lucratif; ce programme visait à rendre accessible la fête populaire aux citoyens du Québec. Si on observe aujourd'hui l'ampleur du phénomène au Québec, on peut conclure que les actions posées ont atteint leurs buts.

La féte populaire s'est imposée comme vếhicule de premier ordre du développement de la culture québécoise, comme activite touristique trés recherchée et comme phénomène économique de plus en plus important. Les contenus des fétes populairés comprennent une diversité d'expressions dans les arts, l'artisanat, les sciences et les techniques.

La Société des festivals populaires du Québec a été créée et un soutien financier progressif lui a été accordé. afin qu èlle puisse regrouper les fètes populaires et que la fête devienne lieu d'expression et de création de la culture populaire québécoise dans ses formes traditionnelles et contemporaines.

\footnotetext{
* Pierre-Paul Leduc est agent de régionalisation et de développement a la Societed des festivals populaires du Quebec.
}

\section{Des centaines de fêtes un peu partout}

A travers le Québec, à toutes les saisons, dans toutes les régions, des fêtes populaires se déroulent. Elles n'ont pas toutes la méme ampleur et certaines sont des eréations récentes.

En 1974, un projet Perspectives Jeunesse a permis de faire un inventaire des fêtes populaires à partir de visites régionales; 78 fêtes populaires furent inventoriées.

Aujourd 'hui, la Société des festivals populaires du Québec compte 153 fêtes membres, réparties dans les quinze régions-loisirs du Québec. Il y a encore beaucoup de recrutement à faire puisque des centaines d'organisations non membres pourraient adhérer au réseau des festivals.

Dans son Bottin des fétes populaires 1986, la Société des festivals populaires du Québec a classifié ses membres dans cinq (5) grandes catégories. Il s'agit de: Arts et traditions (15\%); Carnavals (11\%); Loisir, sports et Plein air (30\%); Promotion d'un produit ressource $(39 \%)$; Western $(5 \%)$.

Les fêtes populaires ont tendance à se multiplier depuis quelques annees; on y retrouve une grande variété de thèmes: l'histoire, les saisons, un produit local ou régional, une industrie, un attrait touristique, un trait particulier de la localité, une activité sportive majeur, etc

Ainsi, le Carnaval-Souvenir de Chicoutimi célèbre chaque année un volet de l'histoire régionale et le Festival du Lait de Coaticook en Estrie veut faire découvrir les bienfaits du lait dont la région est grandement productrice. On pourrait ajouter le Festival de l'Oie Blanche de Montmagny, le Festival de l'Orignal à Val d'Or, le Festival du Bleuet du Lac St-Jean, le Festival de la Crevette de Matane, le Carnaval de la Pátisserie de Disraéli, etc

Ces fêtes suscitent des rassemblements très variables; certaines petites municipalités attirent, par l'originalité de leurs festivités. des participants des régions voisines et vont jusqu'à quintupler leur population. Les fêtes d'envergure nationale ont des chiffres éloquents à ce sujet. Ainsi, dans la région du Saguenay/Lac St-Jean, le CarnavalSouvenir de Chicoutimi a attiré environ 200,000 participants en 1983, tandis que la Huitaine de Gaieté de Roberval en a reçu environ 80,000 en 1982 .

Mème si les fêtes thématiques se déroulent presque toute l'année, plus de la moitié des fêtes de la Société des festivals populaires du Québec ont lieu au cours des mois de juillet et août. Dame Nature y est sûrement pour quelque chose.

Lors des festivités, on y danse, chante et s'amuse pendant des périodes plus ou moins longues allant de deux à dix jours en moyenne. Les participants sont de tous les âges et proviennent de tous les milieux. Ces temps de fêtes permettent à ceux qui vivent quotidiennement séparés par les obligations, le travail et la distance de se retrouver et de participer à des activités communes. La préparation de ces fêtes varie beaucoup selon l'envergure: certaines petites fétes s'organisent en deça de 5 semaines, d'autres exigent des mois de préparatifs et le lendemain de la clôture, il faut déjà penser à la prochaine édition.

Tous les prétextes sont bons pour fêter. On célèbre la jeunesse, le printemps, les sports, le lait, l'oie blanche, les fraises, les framboises... On veut s'amuser, sé récréer, oublier. On weut promouvoir la culture, les loisirs et le tourisme. L'essentiel est de découvrir une bonne raison de fêter et au besoin de l'inventer.

Les objectifs sociaux s'expriment en termes de divertissement populaire et d'animation sociale. Ils semblent traduire assez bien les intérêts manifestés par la population. Les objectifs culturels réferent surtout à la promotion de l'expression culturelle en ces formes. Quant aux objectifs économiques, ils s'énoncent autour de deux póles principaux: l'apport financier et le développement de l'infrastructure économique par le tourisme, l'industrie et d'autres ressources locales.

Certaines fêtes sont nées du désir de renforcement touristique, d'autres sont nees de tentatives d'animation sociale en milieu 


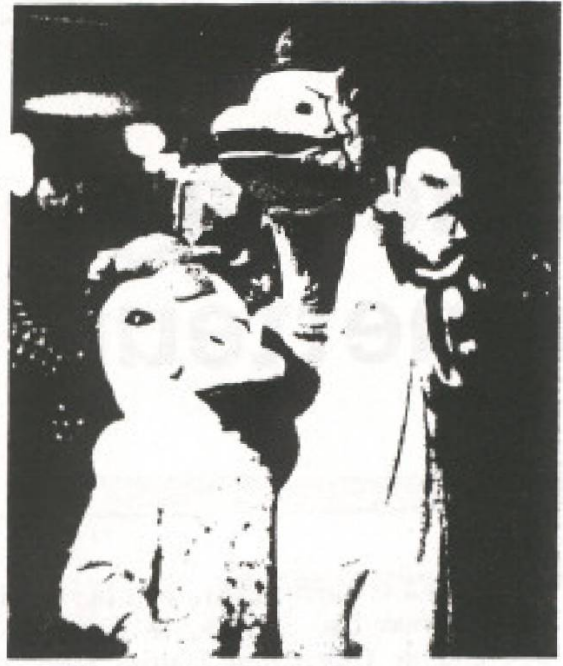

Pan Pan et Pampinot, les personnagesvedettes du Festival de l'Oie Blanche de Montmagny, "Plus que des mascottes... de véritables ambassadeurs"

urbain. Plusieurs fêtes permettent les retrouvailles de gens originaires d'une localité ou de la régión.

La mise sur pied d'une fête populaire est souvent complexe et onereuse. Les promotteurs et organisateurs doivent se donner des modalités de fonctionnement qui déterminent en grande partie le succès de l'événement.

Plusieurs fêtes thématiques s'animent autour d'un bonhomme ou d'un personnage symbolique, d'une mascotte qui sont, en plus d'être les principaux animateurs, des ambassadeurs par excellence. Qui n'a pas entendu parler du célèbre Bonhomme Carnaval à Québec ou de Pépère Joyeux à Chicoutimi. On peut participer à une panoplie d'activites qui vont du concours de recettes, de costumes ou de monuments en passant par des séances de dégustation et les

Tableau 1

Liste d'activités d'une fette

- Messes, bentdictions.

- Concerts, spectacles (artistes bocaux ou rtgionaux, artistes connus)

- Mascarades, bals populaires, soirtes sociales.

- Defiles (vieilles woitures, chars allegoriques, person. mage de marque, etc.)

- Parades (farfares, animgux, chars allegoriques, ete, )

- Marche, promenade.

- Cocktail, degustations, repas gastronomique, piqueniques, tabless, dejeunet des placoteur, toundes des grands ducs, repas troditionnel (cipailles, crèpes, produits de l'terable, feves au lard, soupe aux pois, tourtietes, ragoìt, etc.) repas régionalx (bleuets, poissoc, gourgane, ofe blanche, orignal, gibelote, ete-)

- Exposition, marche, vente trotioir, demonstrations.

- Jeur et conoours, tir de chevaux, tir de tracteurs, sport, rodéo.

- Tournois, bingo, tirage, casino.

- Marcheton, bercethon, dinsethon.

- Partie de sucre, tpluchefte de blé d'inde.

- Rallye, courses, cliniques.

- Activité de plein air, découverte de l'environaement. visites guiders. repas sans oublier les démonstrations et les expositions, le tout centré sur la thématique.

\section{L'avenir de la fête populaire au Québec}

On prévoit cette année une hausse d'environ $30 \%$ des touristes au Québec compte tenu d'un ensemble de facteurs.

À chaque année, les prévisions concernant le nombre de touristes sont des données avec lesquelles les intervenants en tourisme doivent composer. On doit se réjouir des augmentations tout en s'assurant que nous pourrons offrir un produit touristique de plus en plus satisfaisant à ces nombreux visiteurs.

Les organisations de festivals ne font pas exception. Elles ont un produit touristique à offrir et leur réflexion doit tenir compte de la clientèle dans son ensemble. Tout en maintenant leurs caractéristiques propres. branchées sur la réalité d'une ville ou d'une région, les festivals doivent offrir un produit et des commodités susceptibles de combler les attentes des touristes venant de partout. Rien ne doit être laissé au hasard pour attirer cette clientèle friande d'originalité, de qualité et de diversité

Comme nous avons des ressources et des attraits, les festivals dojvent poursuivre leur travail de mise en valeur tout en améliorant leur promotion.

Les fêtes doivent aussi consolider leur organisation, s'assurer de la continuité, de la permanence, tout en tirant profit des compétences disponibles.

Une solution nouvelle et efficace pour éviter l'amateurisme et le temporaire réside sûrement dans le faire faire ou la soustrairance. Déjá, plusieurs festivals y ont recours. Il s'agit de confier l'organisation des événements à des organismes collaborateurs spécialisés, en ayant pris soin de subdiviser les tåches. Ainsi, la publicité peut être confiée à des A.T.R. ou à d'autres organisations ayant une expertise dans le domaine, moyennant une entente financière. Il peut en être ainsi dans tous les autres secteurs de l'organisation.

En fonctionnant de la sorte, la qualité du produit offert sera améliorée (puisque l'on aura fait appel à des gens d'expérience). plus de ressources humaines seront impliquées et la tâche des organisateurs sera réduite et plus facile à assumer. Nos organisateurs de festivals deviendront, dans ces circonstances, des coordonnateurs et ils pourront se réserver certaines tâches précises dans lesquelles ils se sentent plus à l'aise et desquelles ils retirent davantage de satisfaction.

L'avenir passe aussi par la concertation régionale. Les responsables de fétes d'une mềme région doivent se rencontrer, échan- ger leurs expériences, négocier conjointement des contrats (si possible) et surtout planifier leurs actions pour éviter de se nuire mutuellement. Par exemple. l'élaboration d'ur calendrier régional des fétes doit étre encouragée pour éviter le chevau. chement et la concurrence au niveau de la clientèle.

Les relations avec les entreprises privếes nécessitent tegalement beaucoup d'attention. Les échanges doivent être accentués de manière à ce que des projets de développement puissent être mis de l'avant conjointement.

Les projets promotionnels à l'intérieur d'une région ou à l'échelle du Québec doivent être soutenus par l'entreprise. Les idées originales mettant en valeur le réseau des festivals doivent ètre encouragées par ceux qui tirent profit de la santé économique et de la performance de nos festivités populaires. En parallele, on devra susciter des contacts plus fréquents entre les entreprises et les organisateurs de fêtes pour que les gens d'affaires puissent mieux faire connaitre leurs produits et services.

A ce niveau, le congrès annuel de la Société des festivals populaires du Québec a un rôle à jouer. Le congrés de mai 1986, à Tadous. sac, a rapproché encore davantage l'entreprise des organisateurs de fêtes. Cette dimension du congrès, en plus des ateliers de formation et de l'aspect démocratique, devra étre accentuée.

Finalement, l'avenir de la fête populaire au Québec passe par l'informatique et plus spécifiquement la télématique. Les organisations membres de la Société des festivals populaires du Québec doivent ètre mises en contact entre elles par le biais de la télé-informatique pour échanger du courrier et des informations sur leurs activités via un babillard électronique. Des entreprises commerciales, les municipalités et tous les organismes concernés par l'avenir de la féte serajent aussi en mesure de communiquer plus efficacement avec le réseau des festivals.

Il pourrait ètre question d'implanter un système de télé-achat pour faciliter les contacts et la transmission de l'information entre les entreprises et les festivals. Il faudra aussi assurer la présence des festivals dans les banques de données informatiques et permettre la communication et l'accès á de nouvelles sources de renseignements.

Le réseau des festivals populaires québécois constitue un atout de taille pour l'industrie du tourisme au Québec. If y a un potentiel formidable dans ces organisations bénévoles qui ont la responsabilité d'événements de grande importance. Plusieurs défis devront étre relevés et plusieurs projets seront mis de l'avant pour que la fête populaire occupe toute la place qui lui revient. 